\title{
Evaluation of the feasibility of intrapancreatic delivery of drug-loaded microparticles via EUS-guided fine needle injection using a swine model $\square$
}

\section{다)(i) $\odot=$}

\author{
Authors \\ Jennifer Caceres ${ }^{*}, 1$, Maria Munoz-Sagastibelza",2, A. K. M. Nawshad Hossian³ , Jenny Paredes², Kaylene Barrera4, \\ George Mattheolabakis ${ }^{3}$, Laura A. Martello², Shivakumar Vignesh²
}

Institutions

1 Medicine Digestive and Liver Diseases, Columbia University Medical Center, New York, New York, United States

2 SUNY Downstate Medical Center, Department of Medicine, Division of Gastroenterology \& Hepatology, Brooklyn, New York, United States

3 University of Louisiana at Monroe, College of Pharmacy, Monroe, Louisiana, United States

4 SUNY Downstate Medical Center, Department of Surgery, Brooklyn, New York, United States

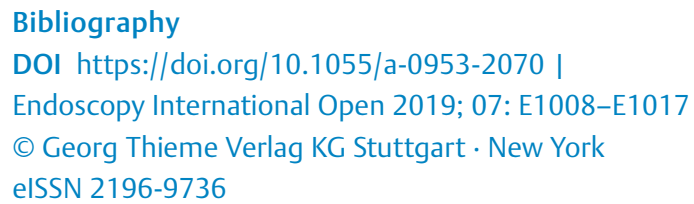

Corresponding author

Shivakumar Vignesh, MD, SUNY Downstate Medical Center, 450 Clarkson Avenue, MSC 1196, Brooklyn, NY 11203, USA Fax: 718-270-7201

shivakumar.vignesh@downstate.edu

\section{ABSTRACT}

Background and study aims Patients with pancreatic cancer often have locally advanced or metastatic disease and are not candidates for curative surgery. Polymer-based microparticles (MPs) represent a drug delivery system that offers sustained release of a chemotherapeutic drug after intralesional injection for local tumor management. The aim of this study was to determine the feasibility of endoscopic ultrasound-guided fine-needle injection (EUS-FNI) of drug-loaded MPs tagged with a fluorophore and fiducial markers for locating the injection site. Secondary aims were to determine the tissue-specific effects of MPs.

Methods Five pigs underwent EUS with selection of an injection site within the pancreas that was marked by placing fiducial markers prior to the MPs injection. EUS-FNI of either blank microparticles (BMPs), containing no drug, or gemcitabine-loaded microparticles (GMPs) was performed. A saline flush containing Spot Endoscopic Marker was used to expel any residual MPs in the needle shaft and tattoo the injection site.

Results A green fluorescent protein flashlight was used to successfully identify the site of MP injection sites in the dissected pancreas. Frozen sections of pig pancreas demonstrated a defined deposit, confirming the delivery of the MPs. Finally, fluorescence microscopy showed activation of caspase-mediated cell death in pancreases of animals that received injections of GMPs.

Conclusions This pilot study demonstrated that fiducial marker placement and pancreatic EUS-FNI of MPs was successful in all pigs with no animals demonstrating pancreatitis. Further studies are needed to determine the role for intralesional injection of drug-loaded MPs in borderline resectable or unresectable pancreatic cancer.

\section{Introduction}

In the United States, pancreatic cancer is the fourth leading cause of cancer deaths and is commonly diagnosed at a late stage [1]. The 5-year survival rate for locoregional disease is estimated at $11.5 \%$ with the 5 -year survival rate of metastatic disease estimated at $2.7 \%$. Approximately 12.5 new cases per 100,000 people per year are diagnosed with pancreatic cancer

\footnotetext{
* Drs. Caceres and Munoz-Sagastibelza: These authors contributed equally.
}

[1]. Several studies have demonstrated the efficacy of chemotherapeutic agents in unresectable disease [2]. In certain patients with locally advanced unresectable disease, gemcitabine remains a viable option in single or combination therapy as a first-line therapy [3-6].

Use of biodegradable polymers, such as poly (lactic-co-glycolic acid) (PLGA), to encapsulate chemotherapeutic agents for direct injection into malignant tumors has been described in prior studies [7]. Drug-loaded microparticles provide sustained intratumoral release of the chemotherapeutic agent 
and lead to less systemic exposure and thus less toxicity [8]. This type of therapy can be considered in patients with borderline resectable disease to convert to surgical candidacy or those with unresectable, locally advanced pancreatic cancer to decrease tumor burden.

Endoscopic ultrasound (EUS) has progressed from existing as a diagnostic tool to serving therapeutic purposes such as fineneedle aspiration and fine-needle injection (FNI) [3,5,9]. EUS has been readily used for diagnosis and staging of gastrointestinal tumors and found to be promising in oncologic therapy, including fiducial placement and direct injection of antitumor agents, given its minimal invasiveness and decreased risk for systemic side effects [5].

\section{Methods}

Five fasted female Yorkshire pigs (weighing approximately 45 to $50 \mathrm{~kg}$ ) were sedated by general anesthesia and endotracheally intubated. A linear array echoendoscope (GF UCT 180) and the Aloka 10 EUS processor were used to perform EUS.

\section{Endoscopic ultrasound-guided fine-needle injection technique}

The entire pancreas was carefully examined. A site within the neck, body or tail of the pig pancreas was selected for EUSguided fine-needle injection (EUS-FNI) of drug-loaded microparticles. FNI was limited to these locations in the pancreas because all pancreatic injections were performed via a trans-gastric approach. Injections were not performed in the head of the pancreas because it was technically more difficult to access the head of the pancreas via a transduodenal approach due to swine anatomy.

An injection site in the pancreas was selected either in the neck, body or tail $(n=4)$ with multiple injection sites selected in pig $5(n=1)$. A 22G fiducial needle was used to deploy a fiducial marker at the location within the pancreas selected for EUSFNI using the standard technique described $[10,11]$. Subse-

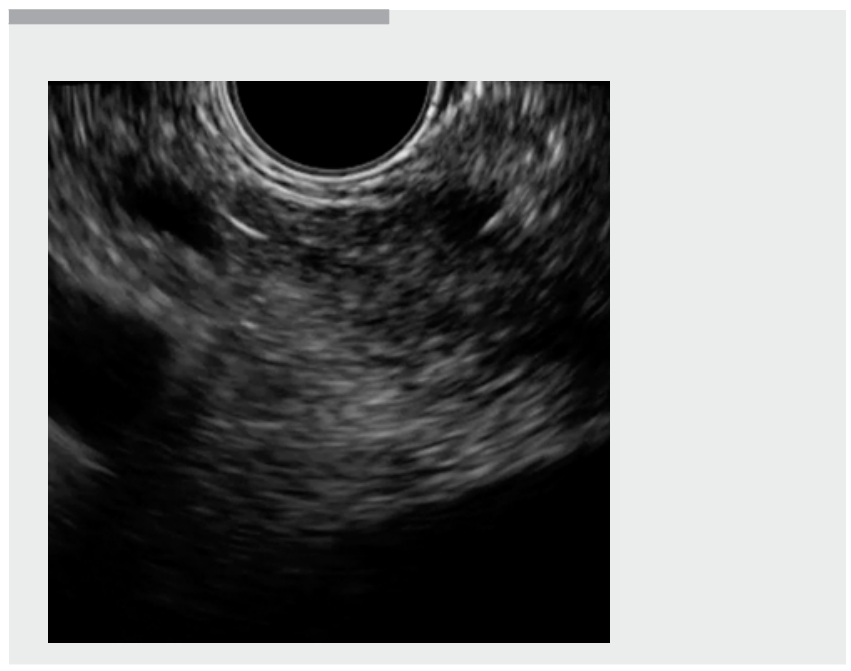

Video 1 EUS video demonstrates placement of a fiducial marker as seen by the hyperechoic focus (pig 3). quently the needle with the fiducial marker was withdrawn from the pancreas and the endoscope. Each injection site was marked with one to two fiducial markers using an EUS-FNA needle preloaded with gold fiducial markers ( $\bullet$ Video 1 ).

A 22G FNA needle was used to perform EUS FNI at the site where the fiducial marker was just placed in all the pigs ( $\triangleright \mathrm{Ta}$ ble 1). A 19G FNA needle also was used for injections in pigs 1 and 4 ( $\triangleright$ Table 1). EUS-FNI of either blank microparticles (BMPs) containing no drug or gemcitabine-loaded microparticles (GMPs) was performed at each site and the nature and volume of the injectate was documented ( $>$ Table 1 ). The injection site was again imaged using linear-array EUS and the needle was carefully advanced through the pancreatic parenchyma under real-time US guidance. To facilitate injection of a viscous solution into solid pancreatic parenchyma, it is essential to create a cylindrical space with the core of the EUS needle and this space is what we called creating a "well" with the needle. After creating the "well," the needle was then slowly withdrawn while the injectate was simultaneously injected in a slow, steady fashion. Power-flow Doppler was turned on prior to injection to visualize and avoid blood vessels in the needle path. A single injection was performed without using the fanning technique. Injection of the microparticles took about 1 minute ( $\triangleright$ Video 2 ).

A solution of normal saline and microparticles was drawn up to make injection volumes of $2 \mathrm{~mL}, 3 \mathrm{~mL}$, and $4 \mathrm{~mL}$. EUS-guided injection of either BMPs or GMPs tagged with fluorophore was then performed anterior or posterior to the fiducial marker based on anatomy ( $\triangleright$ Table $\mathbf{1}$ ). Care was taken to avoid the main pancreatic duct and any major vasculature. A 1-mL saline flush containing Spot Endoscopic Marker was then used to expel any residual microparticles that may have remained in the needle shaft and to tattoo the injection site. Spot Endoscopic Marker is a sterile carbon black suspension that is used for endoscopically tattooing the gastrointestinal tract. This was documented with pictures and videos.

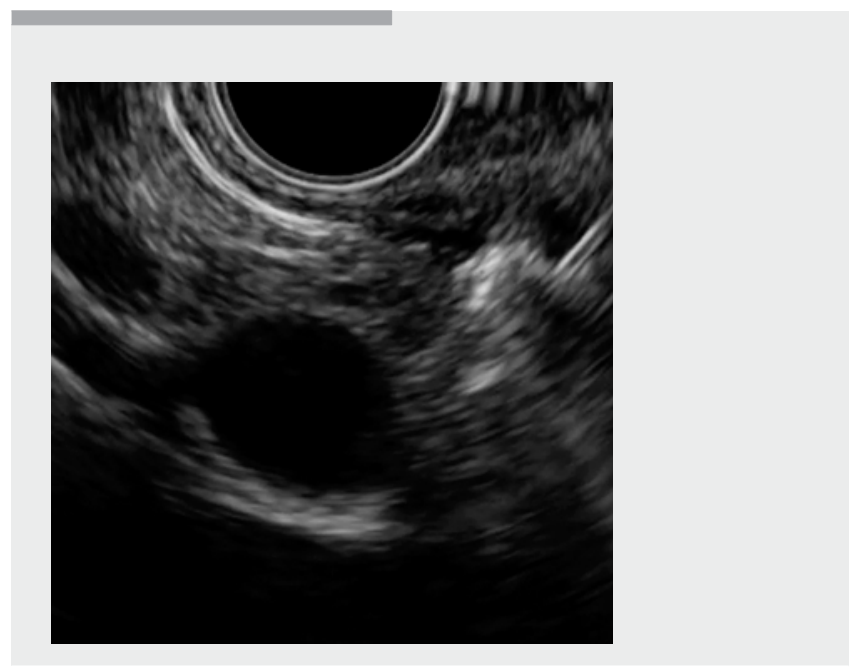

Video 2 EUS video exhibiting injection of the microparticle solution into the pancreas as seen by the anechoic area (pig 1). 
- Table 1 Details of microparticles injections and results of gross examination.

\begin{tabular}{|c|c|c|c|c|c|c|c|c|}
\hline Pig & $\begin{array}{l}\text { Microparti- } \\
\text { cles injected }\end{array}$ & $\begin{array}{l}\text { Volume of } \\
\text { injection }\end{array}$ & $\begin{array}{l}\text { No of fi- } \\
\text { ducials }\end{array}$ & $\begin{array}{l}\text { Site of in- } \\
\text { jection }\end{array}$ & $\begin{array}{l}\text { Size nee- } \\
\text { dle used }\end{array}$ & $\begin{array}{l}\text { Ease of } \\
\text { injection }\end{array}$ & $\begin{array}{l}\text { Compli- } \\
\text { cations }\end{array}$ & Gross examination \\
\hline \multirow[t]{2}{*}{1} & BMP & $2 \mathrm{~mL}$ & 1 & Tail & $19 G$ & Difficult ${ }^{1}$ & None & Fiducial and spot identified, GFP \\
\hline & BMP & $2 \mathrm{~mL}$ & 2 & Body & $22 \mathrm{G}$ & Easy & None & Fiducial and spot not identified \\
\hline \multirow[t]{2}{*}{2} & GMP & $2 \mathrm{~mL}$ & 2 & Tail & $22 \mathrm{G}$ & Easy & None & 2 fiducials identified \\
\hline & GMP & $2 \mathrm{~mL}$ & 2 & Body/neck & $22 \mathrm{G}$ & Easy & None & Nodule at site of injection \\
\hline \multirow[t]{2}{*}{3} & GMP & $3 \mathrm{~mL}$ & 2 & Tail & $22 \mathrm{G}$ & Very easy & None & Fiducials and spot identified \\
\hline & GMP & $3 \mathrm{~mL}$ & 2 & Body/neck & $22 \mathrm{G}$ & Easy & None & Fiducial and spot not identified \\
\hline \multirow[t]{2}{*}{4} & GMP & $4 \mathrm{~mL}$ & 2 & Tail & $22 \mathrm{G}$ & Difficult & None & Nodule at site of injection \\
\hline & GMP & $2 \mathrm{~mL}$ & 2 & Body/neck & $19 G$ & Easy & None & Fiducial and spot not identified \\
\hline 5 & GMP & $\begin{array}{l}2 \mathrm{~mL} \times 3 \text { in- } \\
\text { jections }\end{array}$ & $1,1,1,1^{2}$ & Tail/body & $22 \mathrm{G}$ & $\begin{array}{l}\text { Not re- } \\
\text { corded }\end{array}$ & None & Fiducials and spot identified \\
\hline
\end{tabular}

\section{GMPs and BMPs}

BMPs and GMPs were formulated in the laboratory. Briefly, (PLGA) polymer (Sigma, P1941 75:25) MPs were prepared using $\mathrm{W} / \mathrm{O} / \mathrm{W}$ emulsion technique [12]. To the primary solution, 10 and $20 \mathrm{mg}$ of gemcitabine (Hospira, Inc) were added and the solution was sonicated at three different time settings (42 and 52 seconds and 1 minute) to determine which time setting gives the optimal diameter for the MPs ( 250 microns). Later this solution was added to an external aqueous phase to form the $\mathrm{W} / \mathrm{O} / \mathrm{W}$ emulsion. The MPs were washed three times with sterile phosphate buffered saline. This procedure produced MPs in the range of 200 to 300 microns. The same protocol was followed by the BMPs but water was added instead of gemcitabine.

\section{Detection of apoptosis in pig pancreas}

To detect FITC-MPs, the pancreas was cut, embedded in Optimal Cut Temperature (OCT) and sectioned for immunofluorescence visualization. Slides were fixed with acetone and visualized under a fluorescence microscope. Using the FAM-FLICA kit (SR FLICA Poly Caspase Assay Kit, ImmunoChemistry technologies), we detected polycaspase activation by immunofluorescence in all groups. Briefly, tissue sections were fixed with acetone, stained with FLICA to detect active polycaspases and DAPI to stain the nuclei. A minimum of three independent visual fields were evaluated for each section.

\section{HPLC analysis and drug content measurements for polymeric microparticles}

MPs were lyophilized then analyzed by HPLC. This analysis was conducted using a HITACHI Elite LaChrome HPLC system with UV diode array detector and auto sampler. A total of $20 \mu \mathrm{L}$ of samples were separated using a C18 $4.6 \times 250$-mm 5-micron column at $30{ }^{\circ} \mathrm{C}$ with an isocratic elution starting with $100 \%$
- Table 2 Gemcitabine content in PLGA microparticles as detected by HPLC.

\begin{tabular}{|l|l|l|}
\hline Sample (sonication time) & $\begin{array}{l}\text { \% Drug } \\
\text { loading }\end{array}$ & $\begin{array}{l}\text { \% Encapsulation } \\
\text { efficiency }\end{array}$ \\
\hline GMPs 10 mg Day 0 (45 sec) & 4.31 & 28.78 \\
\hline GMPs 20 mg Day 0 (52 sec) & 1.89 & 12.20 \\
\hline GMPs 10 mg Day 7 (45 sec) & 2.68 & 25.07 \\
\hline GMPs 20 mg Day 7 (52 sec) & 2.01 & 10.05 \\
\hline GMP, gemcitabine-loaded microparticle. & \\
\hline
\end{tabular}

$\mathrm{H}_{2} \mathrm{O}$ for 5 minutes, followed by a gradient towards a volume ratio of 3:17:80 of ACN:MEOH: $\mathrm{H}_{2} \mathrm{O}$ until 12 minutes and maintained this condition for 1 minute, followed by $100 \% \mathrm{H}_{2} \mathrm{O}$ at 14 minutes for 1 additional minute. Flow rate was maintained at $1 \mathrm{~mL} /$ minute throughout the process. GEM was detected at $267 \mathrm{~nm}$, and quantified using a standard calibration curve in the range of 0.05 to $1.00 \mathrm{mg} / \mathrm{mL}$. The percent of drug loading of GEM-loaded PLGA microparticles was quantified using preweighed freeze-dried microparticles dissolved in $1 \mathrm{~mL}$ of DMSO, followed by brief sonication, and analyzed using the aforementioned HPLC protocol. Drug loading was defined as the percentage of the weight of drug detected $(\mathrm{mg})$ by HPLC per weight of microparticles dissolved for the analysis ( $\mathrm{mg}$ ). Encapsulation efficiency was defined as the total drug identified in the formulation, divided by the total drug added (mg) ( $\triangleright$ Table 2 ).

\section{Post-injection phase}

The animals tolerated the procedure well with no immediate adverse events and were subsequently monitored for a total of 7 days post-procedure. Their vitals and feeding habits were monitored as well as any symptoms such as vomiting, de- 

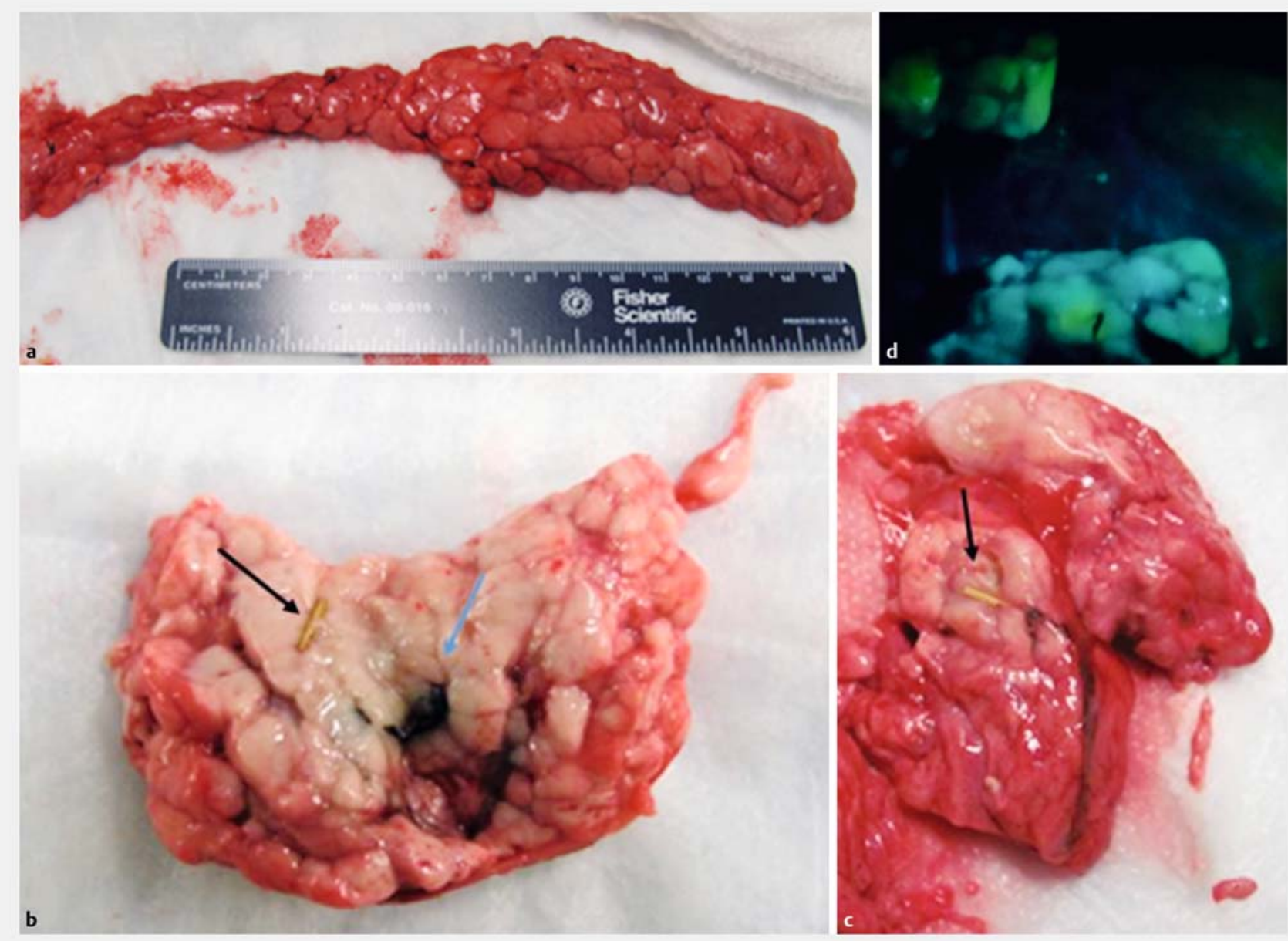

- Fig. 1 Gross examination of pig pancreas after necropsy. a Pig 3 intact pig pancreas. b Pig pancreas demonstrating fiducial marker (black arrow) and spot ink tattoo (blue arrow) in pig 3. c Pig pancreas demonstrating fiducial marker in pig 5). d Green fluorescent protein flashlight shows FITC excitation in BMP injection site. Pig pancreas demonstrated tattoo (site of Spot ink injection) and green fluorescence indicating site of BMP injection in pig 1.

creased activity or abdominal distention. After 7 days, the animals were euthanized and necropsy was performed. The pancreas was successfully harvested from all pigs. After identifying the sites of injection using the fiducial markers and/or the tattoo site, the pancreas was flash frozen for future analysis ( Fig.1).

\section{Results}

\section{Intrapancreatic injection of MPs}

The five Yorkshire pigs underwent EUS - FNI without difficulty and with no complications. A linear hyperechoic focus was noted on EUS upon placement of each fiducial marker ( $\triangleright$ Fig.2). Each animal also demonstrated an intrapancreatic hypoechoic focus after injection of the microparticles solution ( $\bullet$ Fig. 2 ).

Postmortem gross examination of the organs and intra-abdominal cavity was performed ( $\bullet$ Fig. 1 ). The fiducial markers and/or injection site tattoo were identified in four of five pigs. In pig 4, neither a fiducial marker nor a tattoo was identified but a nodule was found within the pancreas indicating the injection site. After necropsy, gross examination with a green fluorescent protein flashlight was also used on each pig pancreas and successfully identified the site of one of four pig pancreases that were injected with fluorescently labeled BMPs (pig 1) $(\triangleright$ Fig. 1). The dissected organs harvested during necropsy were then flash-frozen. Serum lipase levels were measured prior to the procedure for a baseline level and then subsequently at Day 7 prior to euthanasia. There was no elevation in baseline serum lipase levels 7 days post-procedure when compared to the pre-procedure level to indicate onset of pancreatitis.

Pig 1 underwent one injection in the body of the pancreas and one injection in the tail of the pancreas consisting of $2 \mathrm{~mL}$ of BMPs each. A total of three fiducial markers were placed. The decision was made to inject the site anterior or posterior to the fiducial markers depending on anatomy. On gross examination, one of the three fiducial markers was identified as well as the tattooed area containing Spot Endoscopic Marker. ( $\triangleright$ Table 1$)$

Based on the appearance of the fiducial marker on EUS imaging, it was decided to place two fiducial markers in tandem for all of the subsequent injections as this resulted in a prominent 

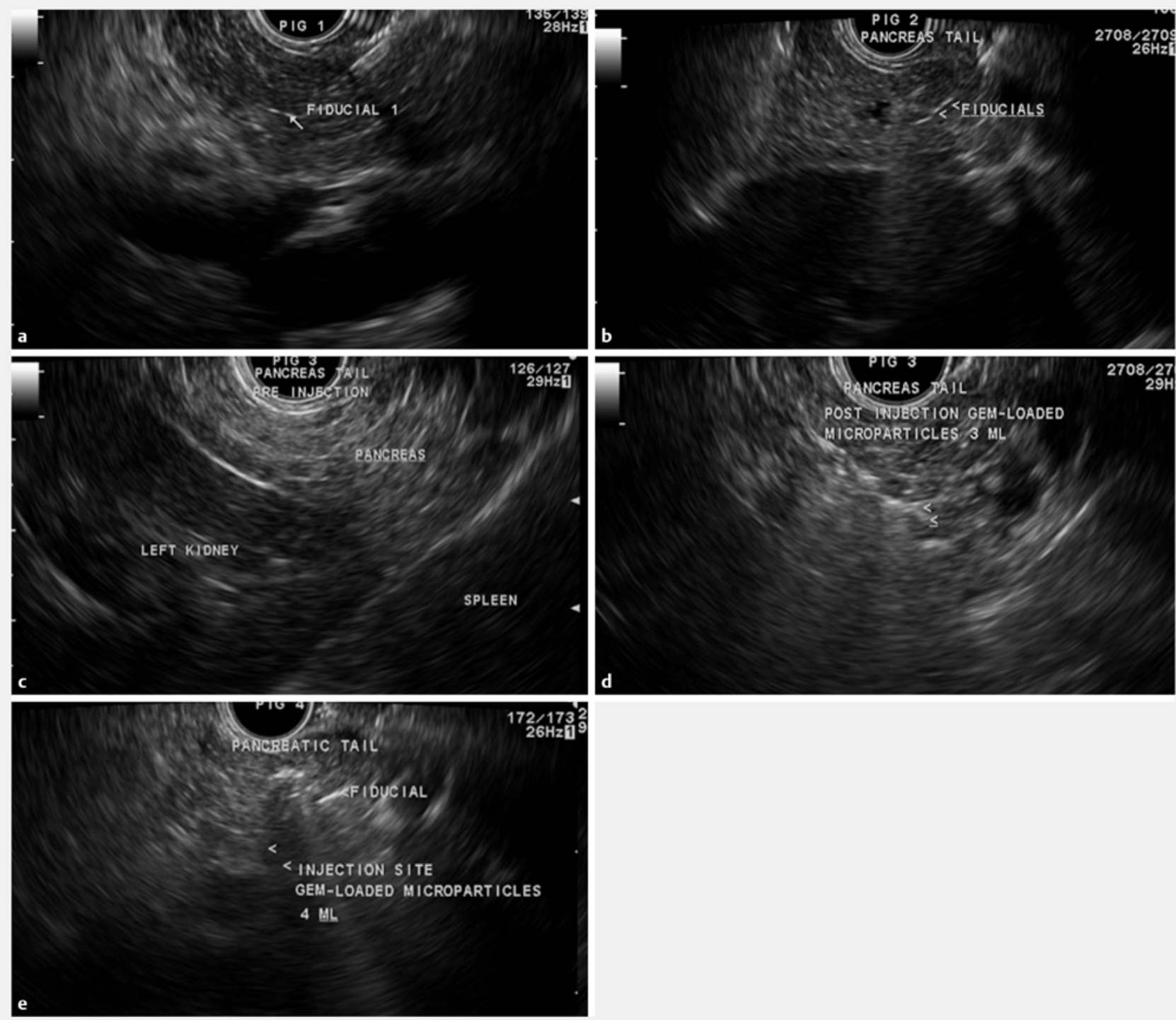

Fig.2 EUS images of fiducial and microparticle injections. The pancreas and surrounding structures were well identified prior to each injection. The fiducial markers are evidenced by an echorich focus in pig $1 \mathbf{a}$ and pig $\mathbf{2} \mathbf{b}$. c This figure demonstrates the pancreas, left kidney and spleen in pig 3. d An anechoic focus denotes the site of the microparticle injection and a hyperechoic strand demonstrates the site of the fiducial marker in pig 3. e The microparticle injection site noted as an anechoic focus in pig 4.

hyperechoic focus, improving visibility of the markers and facilitating subsequent injection of the microparticles ( $\mathbf{F i g . 2}$ ). On gross examination, microparticles deposits were identified within the pig pancreas. The adjacent tissue within the pancreatic tail served as a control for each individual pig to ensure that the microparticles deposit did not migrate inside the pancreas ( Fig.3a, $>$ Fig.3b).

Pig 2 underwent two injections of GMPs consisting of $2 \mathrm{~mL}$ each after placement of a total of four fiducial markers (2 markers were placed in the body of the pancreas and 2 within the tail of the pancreas). On gross examination, the two fiducial markers within the tail of the pancreas were identified. There was a firm nodule identified at the second injection site within the body of the pancreas ( $\triangleright$ Fig. 3a, $\triangleright$ Fig. $3 \mathbf{b}$ ) thereby identifying the second injection site ( $\vee$ Table 1$)$.

Pig 3 underwent two injections with two fiducial markers placed in the body of the pancreas and two in the tail of the pancreas. Subsequently, a solution of $3 \mathrm{~mL}$ of GMPs was injected in the body and an additional $3 \mathrm{~mL}$ was injected in the tail. During gross examination of the tail, the fiducial markers were identified as well as the tattooed area. Pig 4 underwent a 4-mL injection in the tail and a $2-\mathrm{mL}$ injection in the body. A nodule was palpated in the tail of the pancreas of pig 4 indicating the site of the larger injection. Both pigs 3 and 4 showed a deposit of GMPs in the center of the tail. Adjacent tissue was used as a control to evaluate for MP deposit ( $\triangleright$ Fig. 3a). Because the MPs 

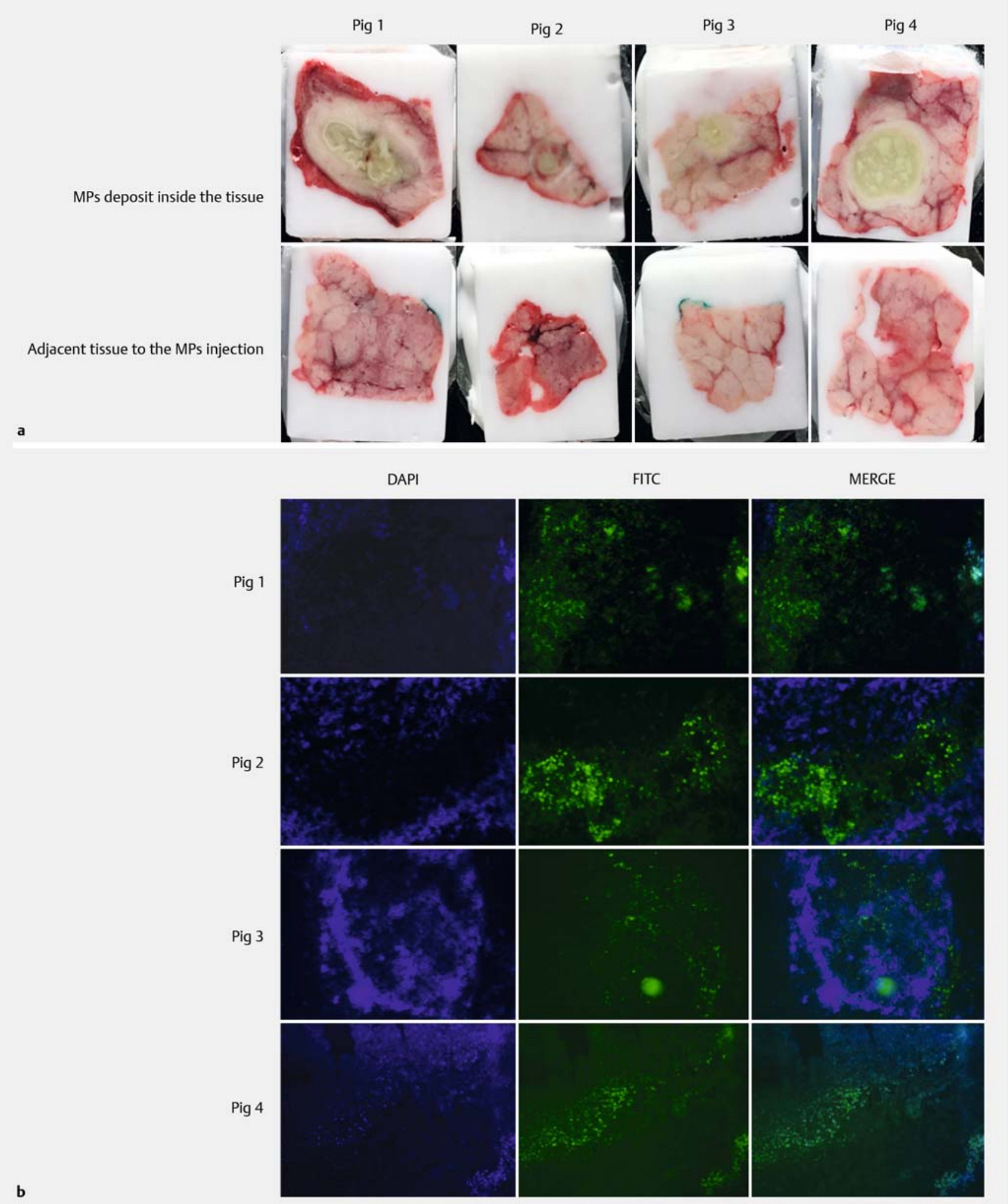

- Fig. 3 Gemcitabine-loaded microparticles deposit as a nodule inside the tissue as shown one week after the injection. a The top set of figures show the four pigs with microparticles deposit inside the pancreatic tissue (green). The lower set of figures were used as controls of the injections, an adjacent sample was taken next to the deposit to show the specific location of the microparticles. $\mathbf{b}$ Areas close to the center where the nodule was detected for each of the pigs using fluorescence microscopy. 
were labeled with FITC, the deposits showed a green/yellow color, which is unique for the MPs ( $\mathbf{F i g . 3 b}$ ).

Lastly, pig 5 underwent three injections of $2 \mathrm{~mL}$ each throughout the tail and body of the pancreas with each injection being approximately 2 -cm apart. When the tail was examined for a deposit, we were not able to localize the MP deposit in the tail of the pancreas. Therefore, pig 5 was excluded from the fluorescence section staining. Pig 5 was the only pig in which a fiducial marker was found outside of the pancreas within the peritoneum.

\section{Detection of apoptosis}

In addition, after identifying the nodule of MPs in the pigs, the tissues were frozen in OCT compound and sectioned $(5 \mu \mathrm{m})$ to visualize FITC-MPs and apoptosis. Each cut was done every 750 microns from one surface to the other and pictures were taken at all levels to detect levels of apoptosis. Because the nodules were localized towards the center of the tissue, we observed activation of apoptosis in this area in the groups injected with GMP (pigs 2, 3 and 4). Adjacent tissue from where the nodule was located was used as control to ensure that the MP deposit stayed within the area of injection. Pig 1 was used as control with BMP and we detected a few cells undergoing apoptosis in the tissue with the nodule or the adjacent tissue ( $\vee$ Fig.4a). In contrast, the tissue sections injected with GMPs displayed groups of cells undergoing apoptosis ( $\mathbf{F i g .} \mathbf{4 b}-\mathbf{d}$ ).

\section{HPLC evaluation}

Using HPLC, we were able to calculate the drug loading (DL) and encapsulation efficiency (EE) for the GMPs. Based on the literature, the DL should be around $2 \%$ to $5 \%$ and the EE around $50 \%$ when using PLGA and gemcitabine. Interestingly, we obtained similar numbers as those published in literature for DL ( $\nabla \mathbf{T a}$ ble 2) [13-16]. Our EE was lower than average, especially in the 20-mg GMPs. This could be attributed to the attempt to dissolve high amounts of drug with a fixed amount of polymer. Interestingly, it appeared that the 10-mg GMPs sonicated at 45 seconds showed a better DL and EE than the ones sonicated with $20 \mathrm{mg}$ of drug at 52 seconds. We believe this phenomenon could be due to variance in sonication time when formulating the MPs. This may have a stronger impact on drug loading than the other parameters. There is potentially an opportunity to improve the $\mathrm{EE}$, although the $\mathrm{DL}$ are as expected in the literature.

\section{Discussion}

In 2018, 55,440 new cases of pancreatic cancer were estimated in the United States with over 44,330 deaths predicted within the same year [17]. Although there has been an improvement in survival rates among most other cancers, the 5-year survival rate among patients with pancreatic cancer has increased modestly from $3 \%$ to $8 \%$. This can be attributed to diagnosing pancreatic cancer at a late stage and the slow advances in developing new treatment modalities.

EUS has proven useful in diagnosis and staging of various gastrointestinal and pancreaticobiliary malignancies. In addi- tion to serving as a diagnostic modality, EUS has evolved into providing oncologic therapeutic options including brachytherapy, celiac plexus neurolysis, fiducial marker placement, EUSguided ablation, and antitumor agent injection [18, 19].

Local tumor therapy has been explored as a viable concept in multimodality therapy of unresectable pancreatic cancer in many pilot studies but failed to demonstrate a survival benefit. There is, however, opportunity to improve the nature of the injectate used and exploring the feasibility of combining it with stereotactic radiation therapy.

EUS-guided fiducial marker placement is performed in patients with locally advanced pancreatic cancer to facilitate stereotactic radiation therapy $[10,11,14,20]$. Our study is the first to attempt fiducial marker placement at the site of EUS-FNI of MPs labeled with fluorophore.

Systemic chemotherapy has been shown to be effective in some patients with advanced unresectable local disease for decreasing tumor burden [8,21,22]. Development of EUS-FNI has expanded the therapeutic role advanced endoscopists can play in treating pancreatic cancer [3,5]. Intratumoral injections of chemotherapeutic agents provide direct tumor therapy with decreased systemic exposure to toxic agents through a minimally invasive procedure. Levy et al. performed a prospective study in which 36 patients with stage II to IV pancreatic cancer underwent intratumoral EUS-FNI with gemcitabine. Among patients with stage III, unresectable disease, four patients were able to be down-staged and subsequently underwent $\mathrm{R} 0$ resection [23].

The literature has described several studies with varying approaches to local therapy for pancreatic cancer. A surgical approach has been described in the past in which Takekuni et al. locally injected a mixture of OK-432 (picibanil), fibrinogen, and thrombin into a patient with unresectable pancreatic cancer and demonstrated a decrease in tumor size and markers [24]. Subsequently, computed tomography (CT)-guided injections were described in the literature. Mulvihill et al. analyzed the safety and feasibility of CT-guided intratumoral injections of ONYX-015, a recombinant adenovirus, in patients with locally advanced pancreatic cancer [25]. Intratumoral pancreatic injection with ONYX-015 and gemcitabine has also been described by Hecht et al. [26]. Other studies have described successful interstitial injections into the pancreas of both swine and canines with no AEs or evidence of pancreatitis $[7,8,27$, 28]. The feasibility of Oncogel injection into the pig pancreas has been demonstrated as has the feasibility of EUS-guided injection of irinotecan-loaded microspheres into the swine pancreas $[7,27,28]$.

In this study, the effects of intrapancreatic injection of BMPs and GMPs into swine pancreas were investigated. This is a novel approach that allows for chemotherapeutic agent encapsulated within the microparticles to be slowly released over time as the microparticles degrade by hydrolysis. Pancreatic cancer has been associated with a significant desmoplastic response which ultimately can create a barrier and limit the amount of drug reaching the tumor when administering systemic chemotherapy. Development and progression of pancreatic cancer are in part attributed to this desmoplastic response $[23,29,30]$. 
Pig 1

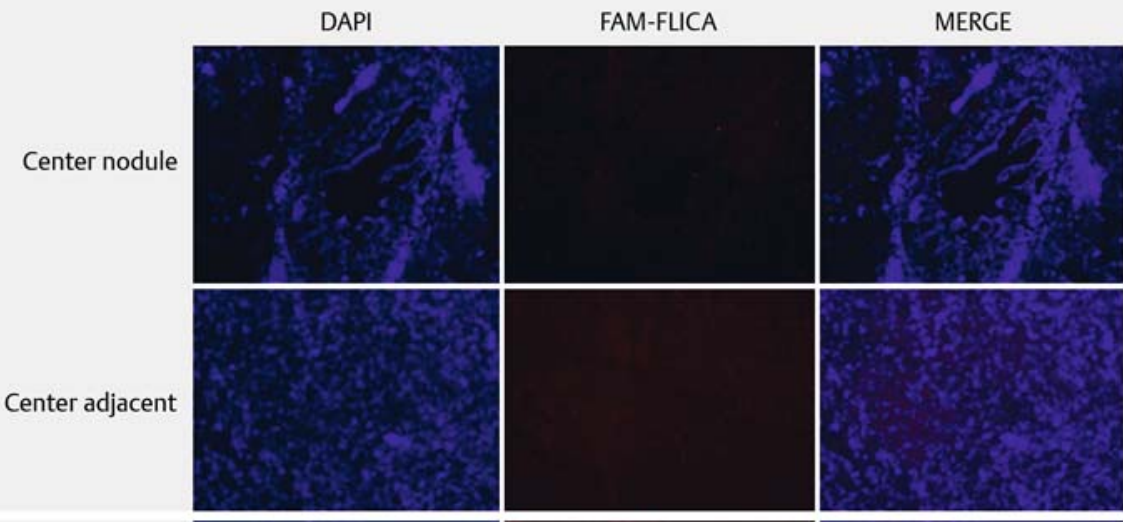

Pig 2

Center nodule
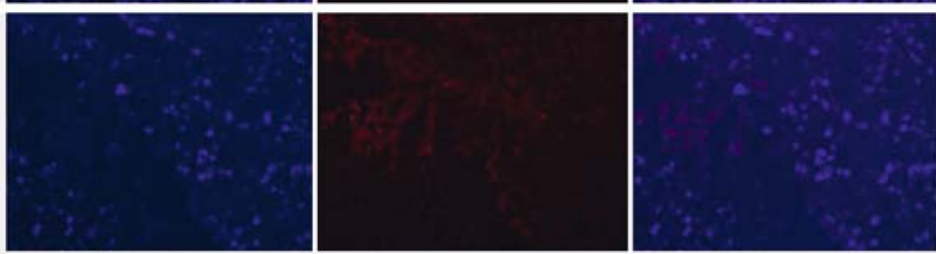

Center adjacent
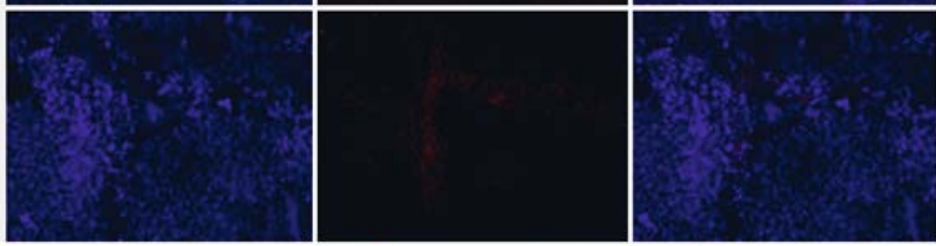

Pig 3

Center nodule
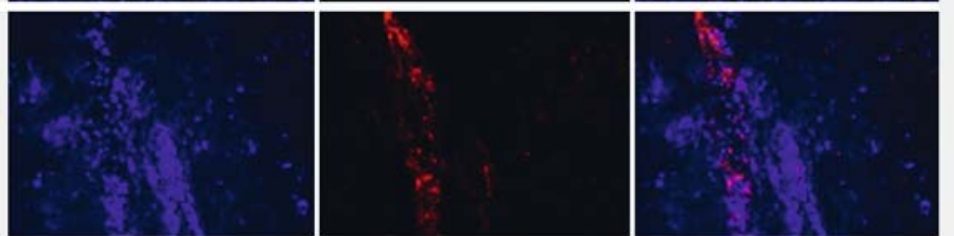

Center adjacent
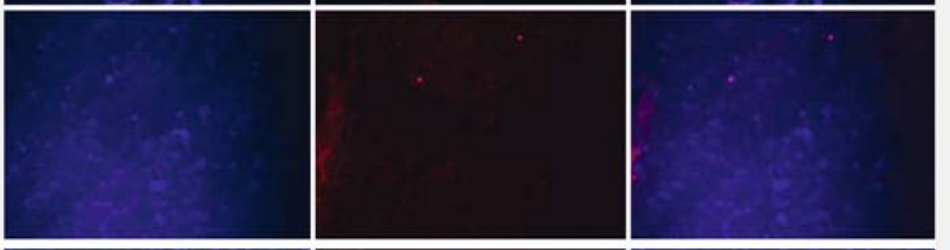

Pig 4

Center nodule
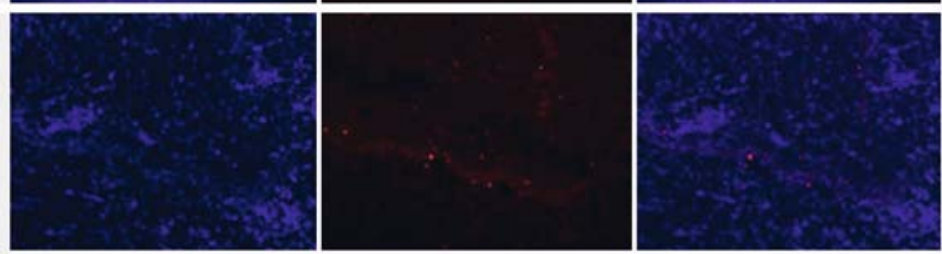

Center adjacent

d

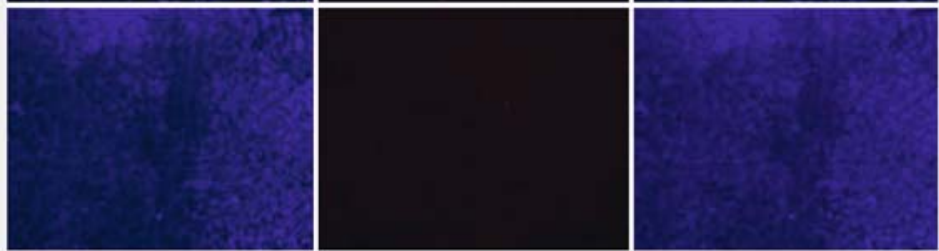

Fig. 4 Activation of apoptosis in pig tissues injected with the drug-loaded microparticles. a One week post-injection, the tail with the 2-mL BMPs deposit with limited caspase-mediated cell death using FAM-FLICA fluorescent reagent (red). The nuclei of the cells were stained with DAPI (blue). The same results are observed in the adjacent control tail tissue. The images were taken close to the center (6,000um depth) of the pancreatic tissue block. b One week post-injection, the neck with the 2-mL GMPs nodule moderately activates caspase-mediated cell death (red) in the surface and the center of the pancreas. The nuclei of the cells were stained with DAPI (blue). As a control, apoptosis was not detected in the adjacent tissue from the neck. The images were taken close the center $(\sim 5,000 \mathrm{um})$ of the tissue block for the site of injection and the adjacent area. c, d The 3-mL and 4-mL deposits, respectively, showed activation of caspase-mediated cell death (red) of groups of cells in the center of the pancreas with the nodule. As a control, apoptosis was not detected in the adjacent tissue from the tail. The images were taken close to the center ( $\sim, 000 \mathrm{um})$ of the tissue block for the site of injection and the adjacent area. All pictures were taken with $10 \times$ magnification. 
There is evidence of heterogeneity and interpatient variability among pancreatic cancers and their response to therapy, which can in part be due to this response [29]. Chemoresistance can therefore occur due to inadequate tumor penetration of the therapeutic agent [23]. Use of drug-loaded MPs has the potential to bypass these limitations.

We successfully injected the pancreas of five pigs and demonstrated that the animals tolerated the procedure well with no post-procedure AEs including no evidence of pancreatitis. Several volumes of injectate were utilized to evaluate whether larger volumes were associated with any evidence of pancreatitis or AEs. There was no evidence of pancreatitis with single injections of $2 \mathrm{~mL}, 3 \mathrm{~mL}$ or $4 \mathrm{~mL}$. Each pig received a cumulative injection volume of $4 \mathrm{~mL}$ to $6 \mathrm{~mL}$ with no histologic evidence of inflammation or clinical or biochemical evidence of pancreatitis.

We also have demonstrated the feasibility and safety of performing EUS-guided fiducial marker placement and EUS-FNI of MPs in the same session. This is relevant to management of locally advanced pancreatic cancer in most institutions in the United States that offer image-guided radiation therapy to these patients to achieve local control and improve resectability. We had planned to inject quantities up to $4 \mathrm{~mL}$ in addition to fiducial marker placement in this pilot study and wanted to select the part of the pancreas that would be conducive to perform the FNI of up to $4 \mathrm{~mL}$ of injectate and the fiducial marker placement. For our porcine study we had available only a therapeutic EUS linear scope to perform EUS-FNI in the pancreas. We felt that a transduodenal approach using a therapeutic echoendoscope might have additional technical challenges that could have confounded our results when taking into consideration swine anatomy. To achieve all the above objectives with the endoscopes available, we felt that the body/neck and tail of the pancreas were the best targets.

We established that we were able to efficiently deliver the microparticles into the pancreas, which was corroborated by deposits or nodules noted on gross examination in the areas where the needles were directed. We used adjacent tissue as controls and we did not detect a nodule or activation of apoptosis outside the areas of injection, indicating that release of drug was confined within the area of injection. In addition, we detected global activation of apoptosis in the pigs that received GMPs and in the areas where the GMP deposits were located.

Analytical studies using HPLC measured the percent drug loading and the percent encapsulation efficiency in the GMP batches. Importantly, we were able to obtain similar numbers for the loading compared to the nanoparticle formulation published in the literature $[13,14,16]$. Sonication is a key parameter in formulation of drug-loaded MPs. We used different sonication times to encapsulate GEM inside the polymer, and the HPLC results suggest that the longer the sonication, the more the $\mathrm{DL}$ is affected. We believe that this factor impacted the values obtained with HPLC. However, these studies demonstrated the feasibility of delivering MPs and detection of apoptosis related to delivery of the drug to the pancreas.

Theoretically, direct injection of drug may only be effective for a short period of time until the drug is taken up by the cells.
Our novel approach demonstrates methods to localize MPs inside the tumor with a daily slow controlled release over a period of weeks without the need for additional injections. We have demonstrated that fiducial markers can be easily placed to localize the injection site and do not cause pancreatitis even with $4 \mathrm{~mL}$ of MPs. Fluorescent-labeled GMPs are another method of localizing injection sites but the dose of fluorophores needs to be studied further.

Pancreatic cancer often requires treatment with a multimodality approach and at times cannot be cured with surgery alone [21]. Targeted treatment with intratumoral injections of a chemotherapeutic agent that can provide sustained release of drug is a novel approach that deserves further investigation. This approach can potentially be offered to patients with borderline resectable disease to downsize tumor and then be considered for surgery or to those with locally advanced unresectable pancreatic cancer. EUS-FNI has the potential to play a pivotal role in the future of targeted cancer therapy. Challenges and potential technical issues addressed in this study included the ease of injection with two different gauge needles in addition to assessing the ease of injection of the solution containing the MPs and demonstrating no AEs.

\section{Conclusion}

In conclusion, we have demonstrated that EUS-FNI is a viable option for intratumoral injection of the pancreas with fiducial markers and varying volumes of drug-loaded MPs with no AEs noted as well as potential efficacy. Our pilot study demonstrates the feasibility and safety of EUS-guided fiducial marker placement combined with EUS-FNI of drug-loaded microparticles in the same session in pigs. Further studies are needed to determine the role for intralesional injection of drug-loaded microparticles in borderline resectable and unresectable pancreatic cancer.

\section{Acknowledgements}

The authors acknowledge the contribution of the Division of Comparative Medicine (DCM) at SUNY Downstate Medical Center and Dr. Montclare's laboratory (Lindsay Hill, Andrew Olsen and Joe Thomas) for use of their lyophilizer machine.

\section{Competing interests}

None

References

[1] National Cancer Institute. SEER Cancer Stat Facts: Pancreas Cancer. Available at https://seer.cancer.gov/statfacts/html/pancreas.html

[2] Kozuch P, Petryk M, Evans A et al. Therapy for regionally unresectable pancreatic cancer. Surg Clin North Am 2001; 81: 691 -697

[3] Ashida R, Chang KJ. Interventional EUS for the treatment of pancreatic cancer. J Hepatobiliary Pancreat Surg 2009; 16: 592 - 597 
[4] Cunningham D, Chau I, Stocken DD et al. Phase III randomized comparison of gemcitabine versus gemcitabine plus capecitabine in patients with advanced pancreatic cancer. J Clin Oncol 2009; 27: 5513 5518

[5] Han J, Chang KJ. Endoscopic Ultrasound-Guided Direct Intervention for Solid Pancreatic Tumors. Clin Endosc 2017; 50: 126 - 137

[6] Noda Y, Goshima S, Kawada H et al. Modified National Comprehensive Cancer Network criteria for assessing resectability of pancreatic ductal adenocarcinoma. AJR Am J Roentgenol 2018; 210: 1252-1258

[7] Karaca C, Cizginer S, Konuk Y et al. Feasibility of EUS-guided injection of irinotecan-loaded microspheres into the swine pancreas. Gastrointest Endosc 2011; 73: $603-606$

[8] Sun S, Wang S, Ge N et al. Endoscopic ultrasound-guided interstitial chemotherapy in the pancreas: results in a canine model. Endoscopy 2007; 39: $530-534$

[9] Seo DW. EUS-guided antitumor therapy for pancreatic tumors. Gut Liver 2010; 4: (Suppl. 01): S76-81

[10] Davila Fajardo R, Lekkerkerker S], van der Horst A et al. EUS-guided fiducial markers placement with a 22-gauge needle for image-guided radiation therapy in pancreatic cancer. Gastrointest Endosc 2014; 79: $851-855$

[11] Varadarajulu S, Trevino JM, Shen S et al. The use of endoscopic ultrasound-guided gold markers in image-guided radiation therapy of pancreatic cancers: a case series. Endoscopy 2010; 42: 423-425

[12] Varde NK, Pack DW. Microspheres for controlled release drug delivery. Expert Opin Biol Ther 2004; 4: 35-51

[13] Danhier F, Ansorena E, Silva JM et al. PLGA-based nanoparticles: an overview of biomedical applications. J Control Release 2012; 161: $505-522$

[14] Yu X, Di Y, Xie C et al. An in vitro and in vivo study of gemcitabineloaded albumin nanoparticles in a pancreatic cancer cell line. Int J Nanomedicine 2015; 10: 6825-6834

[15] Silva AL, Rosalia RA, Sazak A et al. Optimization of encapsulation of a synthetic long peptide in PLGA nanoparticles: low-burst release is crucial for efficient CD8(+) T cell activation. Eur J Pharm Biopharm 2013; 83: $338-345$

[16] Yang YY, Chung TS, Ng NP. Morphology, drug distribution, and in vitro release profiles of biodegradable polymeric microspheres containing protein fabricated by double-emulsion solvent extraction/evaporation method. Biomaterials 2001; 22: 231-241

[17] American Cancer Society. Cancer Facts and Figures. Available at https://www.cancer.org/research/cancer-facts-statistics/all-cancerfacts-figures/cancer-facts-figures-2018.html
[18] Kaplan ], Khalid A, Cosgrove $\mathrm{N}$ et al. Endoscopic ultrasound-fine needle injection for oncological therapy. World J Gastrointest Oncol 2015; 7: $466-472$

[19] Verna EC, Dhar V. Endoscopic ultrasound-guided fine needle injection for cancer therapy: the evolving role of therapeutic endoscopic ultrasound. Therap Adv Gastroenterol 2008; 1: $103-109$

[20] Sanders MK, Moser AJ, Khalid A et al. EUS-guided fiducial placement for stereotactic body radiotherapy in locally advanced and recurrent pancreatic cancer. Gastrointest Endosc 2010; 71: 1178-1184

[21] Bergenfeldt M, Albertsson M. Current state of adjuvant therapy in resected pancreatic adenocarcinoma. Acta Oncol 2006; 45: 124-135

[22] Wilkowski R, Thoma M, Bruns C et al. Chemoradiotherapy with gemcitabine and continuous 5-FU in patients with primary inoperable pancreatic cancer. JOP 2006; 7: 349-360

[23] Levy M], Alberts SR, Bamlet WR et al. EUS-guided fine-needle injection of gemcitabine for locally advanced and metastatic pancreatic cancer. Gastrointest Endosc 2017; 86: 161 - 169

[24] Takekuni K, Sakon M, Kishimoto S et al. [A patient with unresectable cancer of pancreas head, effectively treated by a local injection of the mixture of OK-432, fibrinogen and thrombin]. Gan To Kagaku Ryoho 1996; 23: $1621-1623$

[25] Mulvihill S, Warren R, Venook A et al. Safety and feasibility of injection with an E1B-55 kDa gene-deleted, replication-selective adenovirus (ONYX-015) into primary carcinomas of the pancreas: a phase I trial. Gene Ther 2001; 8: 308-315

[26] Hecht JR, Bedford R, Abbruzzese JL et al. A phase I/II trial of intratumoral endoscopic ultrasound injection of ONYX-015 with intravenous gemcitabine in unresectable pancreatic carcinoma. Clin Cancer Res 2003; 9: 555-561

[27] Linghu E, Matthes K, Mino-Kenudson M et al. Feasibility of endoscopic ultrasound-guided OncoGel (ReGel/paclitaxel) injection into the pancreas in pigs. Endoscopy 2005; 37: 1140-1142

[28] Matthes K, Mino-Kenudson M, Sahani DV et al. EUS-guided injection of paclitaxel (OncoGel) provides therapeutic drug concentrations in the porcine pancreas (with video). Gastrointest Endosc 2007; 65: $448-453$

[29] Koay E], Baio FE, Ondari A et al. Intra-tumoral heterogeneity of gemcitabine delivery and mass transport in human pancreatic cancer. Phys Biol 2014; 11: 065002

[30] Neesse A, Michl P, Frese KK et al. Stromal biology and therapy in pancreatic cancer. Gut 2011; 60: $861-868$ 